\title{
ACKNOWLEDGEMENT TO REFEREES
}

(C) Springer International Publishing Switzerland 2016

Dear Reader,

As we approach the end of 2016, and publish our final issue of Applied Health Economics and Health Policy for the year, I wish to reflect on another successful year for the journal and for others in the Adis Premier journals portfolio, and to thank all who have contributed to Applied Health Economics and Health Policy over the past 12 months.

The Adis journals portfolio will expand in 2017, with the launch of PharmacoEconomics - Open. This new journal will be fully open access and will focus on the publication of applied research on the economic implications and health outcomes associated with drugs, devices and other healthcare interventions. It joins our other well respected health economics and outcomes research publications, PharmacoEconomics, Applied Health Economics and Health Policy, and The Patient: Patient-Centered Outcomes Research.

Another important milestone was reached for our recently launched open access journals, Drugs - Real World Outcomes and Drug Safety - Case Reports. Content from these journals is now available on PubMed Central.

I offer my sincere thanks to all authors who have contributed articles to Applied Health Economics and Health Policy over the course of 2016. Their skill and dedication are critical to the continued publication of the journal. The quality of published articles is, similarly, testament to the significant efforts of the peer reviewers, whose commitment ensures that the journal's content is held to the highest possible standard. I would like to thank the following individuals who acted as reviewers for Applied Health Economics and Health Policy in the last 12 months:

Ivo Abraham, USA

Fernando Antonanzas Villar, $*$ Spain

Darrin Baines, UK

Azad Bali, * Australia

Konstantin Beck, Switzerland

Jonathan D. Belsey, UK

Esther Bettiol, Switzerland

Joke Bilcke, Belgium

Jeffrey S. Borer, USA

Karen Bremner, Canada

Ethel Brinda, Denmark

Valentin Brodszky, Hungary

Josh J. Carlson, USA

James D. Chambers, USA

Harrell W. Chesson, USA

Andrew Clark, UK

Justin M. Cohen, USA

Daniel Collado-Mateo, Spain

Merce Comas, * Spain
William H. Crown, USA

Jeffrey R. Curtis, USA

Alessandro Curto, Italy

Theodore E. Day, USA

Mario de Lemos, Canada

Anne Dee, Ireland

Manuela Deidda ${ }^{\dagger}$ Italy

Benoit Dervaux, France

Vakaramoko Diaby, USA

Brett Doble, Australia

James Dolan, USA

Flávia Tavares Silva Elias, Brazil

Gihan Elsisi, Egypt

Yu Fang, China

Denzil G. Fiebig, Australia

Richard G. Fiscella, USA

Jennifer Flynn, Canada

Heather Foulds, Canada

Vassilis Fragoulakis, Greece 
Gerardus W.J. Frederix, The Netherlands

Chien-Hao Fu, Taiwan

Róbert Gál, Hungary

Michael Ganz, USA

Patrick P. Gleason, USA

Brian G. Godman, Sweden

Elizabeth Goodwin, UK

J. Ross Graham, Canada

Scott D. Grosse, USA

Indrani Gupta, India

Samer Hamidi, United Arab Emirates

Stephen Harbarth, Sweden

Hideki Hashimoto, Japan

Alex Jingwei He, Hong Kong

Eveline A. Heijnsdijk, The Netherlands

Vivian Ho, USA

Rumana Huque, Bangladesh

Don Husereau, Canada

Sergio Iannazzo, ${ }^{\dagger}$ Italy

Yawen Jiang, USA

Barbara H. Johnson, USA

Bengt Jonsson, Sweden

Zoltan Kalo, Hungary

Papar Kananurak, Thailand

Anuraag Kansal, USA

Andreas Karabis, The Netherlands

Anup Karan, India

Pawel Kawalec, Poland

Brandon C. Koford, USA

Rositsa Koleva-Kolarova, UK

Finn B. Kristensen, Denmark

Hans Krueger, Canada

Kaushalendra Kumar, India

Janice Kwon, USA

Allen Lai, Singapore

Mark M. Lamotte, Belgium

Reiner Leidl, Germany

Andrew J. Leidner, USA

Carol Levin, USA

Emmy Y. Li, China

Nicola Lucio Liberato, Italy

Frank R. Lichtenberg, USA

Robert Likic, Croatia

Andrew Lloyd, UK

Alia Gonzales Luz, Thailand

Jared Lane K. Maeda, USA

Pierre-Alexandre Mahieu, France

Laxmaiah Manchikanti, USA

Paolo Mancuso, Italy

Danijela Maras, Canada

Andrea Marcellusi, Italy

Joachim Marti, Switzerland

Andrew Mason, USA
Leonardo J. Mastronardi, Argentina

Céu Mateus, Portugal

Josephine A. Mauskopf, USA

Robin McKnight, USA

Elizabeth Mearns, USA

Ricard Meneu, Spain

Carine Milcent, France

Sepideh Modrek, USA

Chantal Morel, UK

Haizhen Mou, Canada

Dirk Mueller, Germany

Nikolai Mühlberger, Austria

Brendan Mulhern, Australia

Daniel Murphy, UK

Narayana Muttur Ranganathan, India

Anthony T. Newall, Australia

Austin Nichols, USA

Justice Novignon, Ghana

Katie Page, Australia

K. T. Park, USA

Mike Paulden, Canada

Kim Pauwels, Belgium

Alison Mary Pearce, Australia

Sandra Peer, Austria

Ulf Persson, Sweden

Richard Peter, The Netherlands

Richard Pitman, UK

Laura T. Pizzi, USA

Daniel Pollard, UK

Judi Porter, Australia

Maarten Postma, The Netherlands

Jiwei Qian, Singapore

$M$ Ramesh, Singapore

Julie Ratcliffe, Australia

Ramin Ravangard, Iran

Cécile Rémuzat, France

Bjarne Robberstad, Norway

Michael D. Rosko, USA

Joan Rovira, Spain

Carlos Rubio-Terres, Spain

Lewis Ruff, UK

Zia Sadique, UK

Erik Schokkaert, Belgium

Christian Selinger, UK

Asrul Akmal Bin Shafie, Malaysia

Steven Sheingold, USA

Ji-Tian Sheu, Taiwan

James Signorovitch, USA

Sandra F. Simmons, USA

Kit Nordbo Simpson, USA

Anthony So, USA

Steffan Frosi Stella, Brazil

Andrew Stoddart, * UK 
Seher Sülkü, Turkey

Arnela Suman, The Netherlands

Tomoyuki Takura, Japan

Paul Tappenden, UK

Hiromi Terawaki, USA

Dominic Thorrington, UK

Martin Trombetta, Argentina

Antonio Jose Trujillo, USA

Aki Tsuchiya, UK

Charalampos Tzanetakos, Greece

Laura Vallejo-Torres, Spain

Richard van Kleef, The Netherlands
Sukumar Vellakkal, India

Jian Wang, China

Jurgen Wasem, Germany

Jean-Blaise Wasserfallen, Switzerland

Erik L. Werner, Norway

Chapin White, USA

Vera Winter, Germany

Beth Woods, UK

Robert Yates, UK

Kai Yeung, USA

Jongsay Yong, Australia

Richard Zur, Canada

* reviewed 2 manuscripts

${ }^{\dagger}$ reviewed 3 manuscripts

In addition, I would like to thank the members of the journal's Honorary Editorial Board, who have acted as peer reviewers and authors, and have provided guidance on journal content, policy and processes:

John Ataguba, South Africa

Stephen Beard, UK

John Brazier, UK

Chin-Shyan Chen, Taiwan

Chris Doran, Australia

Talitha Feenstra, The Netherlands

Afschin Gandjour, Germany

Livio Garattini, Italy

Denis Getsios, USA

Colin Green, UK

Dan Greenberg, Israel

Hossein Haji Ali Afzali, Australia

Fred Hellinger, USA

Naoki Ikegami, Japan

Michael Iskedjian, Canada

Jonathan Karnon, Australia

Jahangir Khan, UK
Nikos Maniadakis, Greece

Logan McLeod, Canada

Brita Pekarsky, Australia

Jaume Puig-Junoy, Spain

Ken Redekop, The Netherlands

Jordana Schmier, USA

Mark Sculpher, UK

Catherine Sermet, France

Steven Simoens, Belgium

Tom Stargardt, Germany

Lotte Steuten, USA

Sandra L. Tunis, USA

Albert Wertheimer, USA

Jennifer Whitty, UK

Edward Wilson, UK

Bong-Min Yang, South Korea

I hope that you have found the articles published throughout 2016 to be both interesting and informative. I have appreciated the high quality of content contributed to the journal this year and I look forward to keeping you up to date with topical issues in the field in 2017.

With best wishes from

Tim Wrightson

Editor

Applied Health Economics and Health Policy 